The Astrophysical Journal, Vol. 156, June 1969

(C) 1969. The University of Chicago. All rights reserved. Printed in U.S.A.

\title{
INFRARED AND OPTICAL MEASUREMENTS OF THE CRAB PULSAR NP 0532*
}

\author{
G. Neugebauer, $\nmid$ E. E. Becklin, $\ddagger$ J. Kristian,$\dagger$ R. B. Leighton,$\dagger$ \\ G. SNELlen, $\ddagger$ and J. A. Westphal $\dagger$ \\ Mount Wilson and Palomar Observatories, Carnegie Institution of Washington, \\ and California Institute of Technology \\ Received A pril 28, 1969; revised May 8, 1969
}

\begin{abstract}
Observations of the pulsating component of NP 0532 at 2.2 and $1.65 \mu$ are given. The energy density per pulse at $2.2 \mu$ is $(3.2 \pm 0.4) \times 10^{-31} \mathrm{~J} \mathrm{~m}^{-2} \mathrm{~Hz}^{-1}$ and forms a smooth continuation of the visual data.

Shortly after the radio pulsar NP 0532 in the Crab Nebula was identified with an optical object by Cocke, Disney, and Taylor (1969), the absolute spectral-energy distribution was measured by Oke (1969) between the wavelengths of 0.34 and $0.83 \mu$ and was found to be relatively flat. The average energy density in the main pulse was approximately $1.5 \times 10^{-30} \mathrm{~J} \mathrm{~m}^{-2} \mathrm{~Hz}^{-1}$. Oke's measurements were made on three nights; although the intensity varied by $0.5 \mathrm{mag}$, the spectral shape remained unchanged. Comella et al. (1969) have measured the energy density in the pulse at $150 \mathrm{~cm}$ and find it higher than that in the optical by a factor of approximately $10^{3}$. They also find a spectral index $a\left(f_{\nu} \propto \nu^{-a}\right)$ of +2 ; this slope is consistent with an upper limit of $7 \times 10^{-29} \mathrm{~J} \mathrm{~m}^{-1} \mathrm{~Hz}^{-1}$ placed on the energy in the pulse at $13 \mathrm{~cm}$ by Ekers and Moffet (1969). This Letter describes measurements of the pulsar at 2.2, 1.65, and $0.55 \mu$. Although the results are preliminary, the current interest in the energy distribution of pulsars seems to warrant publication at this time.
\end{abstract}

\section{OBSERVATIONS}

The observations of NP 0532 were made between 1900 and 2015 P.S.T. on March 14, 1969, using the infrared photometer described by Becklin and Neugebauer (1968), at the Cassegrain focus of the 200-inch Hale telescope with a focal-plane aperture 7".5 in diameter. The output of the $\mathrm{PbS}$ detector was a.c. amplified and analyzed with a 1024-channel signal-averager set to enhance signals with a period twice the radio and optical period of NP 0532 at the time of observation. The contents of the averager were displayed on an oscilloscope and photographed for later analysis. A digital tape recording of the output signal from the a.c. amplifier was also obtained, but only the results from the averager were used in this reduction.

Two 10-min observations were made at $2.2 \mu$ and one 10-min and one 5-min observation at $1.65 \mu$. Calibration data were obtained by mechanically chopping the light from $\zeta$ Tauri with an asymmetric chopper designed to admit a 1.5-msec pulse of radiation every $38 \mathrm{msec}$. This star has a magnitude at $2.2 \mu$ of 2.94 (Johnson 1964), corresponding to a flux density of $4.2 \times 10^{-25} \mathrm{~W} \mathrm{~m}^{-2} \mathrm{~Hz}^{-1}$; at $1.65 \mu$, its flux density, based on comparisons with a Lyrae, is $6.0 \times 10^{-25} \mathrm{~W} \mathrm{~m}^{-2} \mathrm{~Hz}^{-1}$.

* This work was supported in part by National Aeronautics and Space Administration grant NGL 05-002-007.

$\dagger$ Mount Wilson and Palomar Observatories, Carnegie Institution of Washington, and California Institute of Technology.

$\ddagger$ California Institute of Technology. 
Following the infrared measurements, observations in the $V$-band were made, using the 200-inch prime-focus photometer; the data were again processed by the averager. The instrumentation is similar to that described by Westphal et al. (1969).

The reduction of the infrared data was made difficult by the low signal-to-noise ratio and by the 27 -msec time constant of the $\mathrm{PbS}$ detector, which greatly exceeds the width of the pulse and closely approaches the period of the pulsar. The measurements in the visible showed a main pulse of $1.5 \mathrm{msec}$ full width at half-maximum, followed after 13.5 msec by a secondary pulse of width $3.0 \mathrm{msec}$, which contains approximately half the energy of the main pulse. These are similar to earlier measurements by Lynds, Maran, and Trumbo (1969) and by Nather, Warner, and Macfarlane (1969) and to other unpublished 200-inch measurements. In the reduction it was assumed that the infrared pulses are similar to the visual pulses. The averaged data were compared with the computed

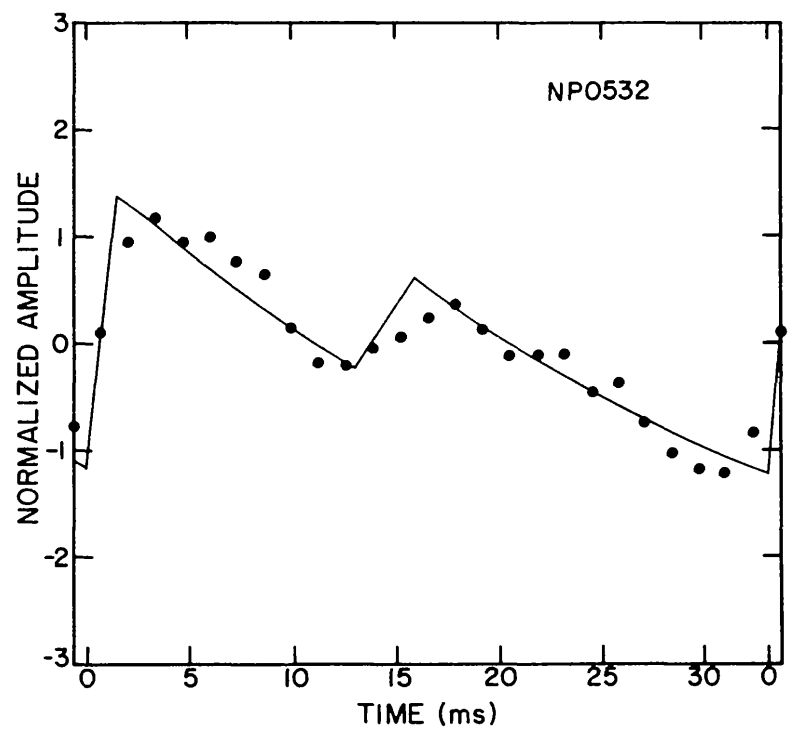

Fig. 1.-Measurement of periodic signals from NP 0532 at $2.2 \mu$ on March 14, 1969, using a $\mathrm{PbS}$ detector with a $27-\mathrm{msec}$ time constant. Points are measured values of signal from the pulsar, averaged for 20 min at the known radio and visual frequency. Solid line shows expected values for a pulsed source with the same characteristics as those observed in the visual. Both main and secondary pulses can be seen.

response of the detector to rectangular input pulses whose widths and relative areas matched those of the visual pulses; their phase and amplitude were varied to obtain the best least-squares fit to the data. The data and the fit at $2.2 \mu$ are shown in Figure 1 . The statistical accuracy of the amplitude is $\sim 5$ per cent at $2.2 \mu$ and $\sim 8$ per cent at $1.65 \mu$. The sensitivity to a shift in phase is quite sharp. The error in the calibration is approximately 10 per cent.

Although the characteristics of the pulses in the visual were used to compute the energy in the main pulse, the calculated energy in the main pulse was found to be not very sensitive to reasonable variations of the assumed widths and relative amplitudes of the pulse and subpulse. For example, when the width of the main pulse was increased by a factor of 3 , the derived value for the energy in the pulse was increased by less than 10 per cent. This means that the calculated value for the energy of the pulse is not likely to be greatly in error, but it does not directly confirm the assumed similarity of the visual and infrared pulse shapes. The ratio of the energy in the secondary pulse to that 
in the primary was found from the infrared data to be $0.43 \pm 0.07$. This measurement is thus consistent with the assumption that the properties of the pulses are the same in the visual and infrared.

In addition to measurements of the pulsar, measurements of the Crab Nebula itself were made at a location approximately $5^{\prime \prime}$ east of the pulsar in order to extend the energy distribution measured there by Oke (1969). Observations were made with the Hooker 100-inch telescope against a sky reference outside the nebula. Over a range of

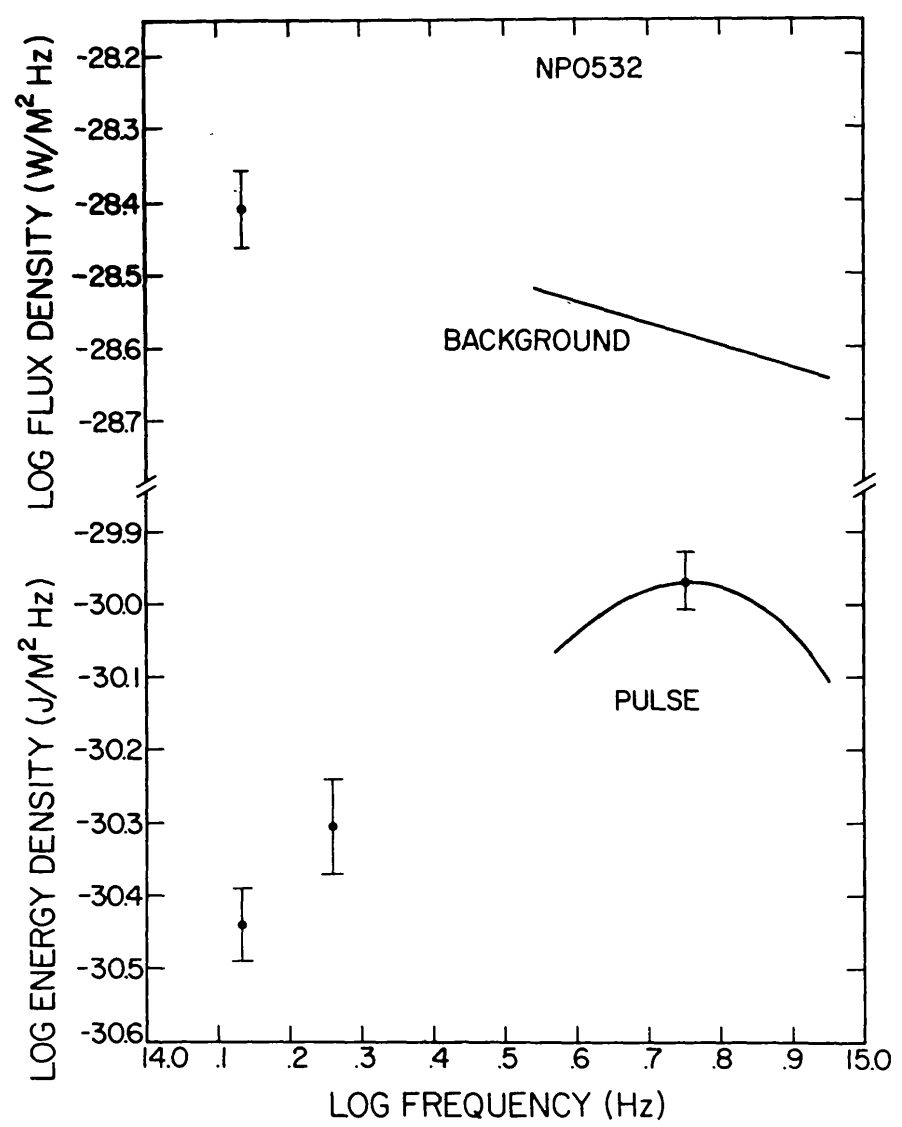

Fig. 2.-Optical energy distribution of the main pulse of NP 0532. Two points at left are measurements at 2.2 and $1.65 \mu$ described in this Letter. Solid line is Oke's (1969) energy distribution, normalized to a measurement made in the $V$-band, one-half hour after the infrared measurements. Positive slope in the near-infrared suggested by Oke's measurements continues out to $2.2 \mu$. Also shown, in the upper part of the figure, is the background flux density within a 3"6 aperture near the pulsar at $2.2 \mu$ and in the visual (Oke 1969).

apertures from $10^{\prime \prime}$ to $45^{\prime \prime}$ the observed flux density per unit solid angle remained constant; the data were therefore compared directly with the data of Oke by normalizing to his aperture of 3 ".6. Absorption corrections of 0.29 mag at $1.65 \mu$ and 0.16 mag at $2.2 \mu$ were made, based on an assumed absorption of 1.7 mag in the visual (Oke 1969) and a reddening curve similar to that of Johnson (1968).

The energy density in the main pulse at $0.55,1.65$, and $2.2 \mu$ and of the nebular flux density at $2.2 \mu$ in a 3".6-diameter aperture is plotted in Figure 2, along with the data of Oke (1969); Oke's data for the pulse, corrected for 1.7-mag absorption, have been adjusted to $V=16.4 \mathrm{mag}$, the value found on the night of the infrared observations. 


\section{DISCUSSION}

The infrared energy densities both for the main pulse and for the nebula fit smoothly on to the visual spectra measured by Oke (1969). The two energy distributions clearly differ and indicate that the detailed origin of the radiation from the pulsar is different from that of the Crab Nebula as a whole; the latter is generally thought to be synchrotron radiation over the entire spectral range. The similarity between the observed spectralenergy distribution of the pulses and that of a $10000^{\circ} \mathrm{K}$ Planck function, noted by Oke, also continues to hold. If, however, the pulsar is located within the Crab Nebula at a distance of $1700 \mathrm{pc}$ and if the dimensions of the pulsar are typically ( $c \times$ pulse duration),

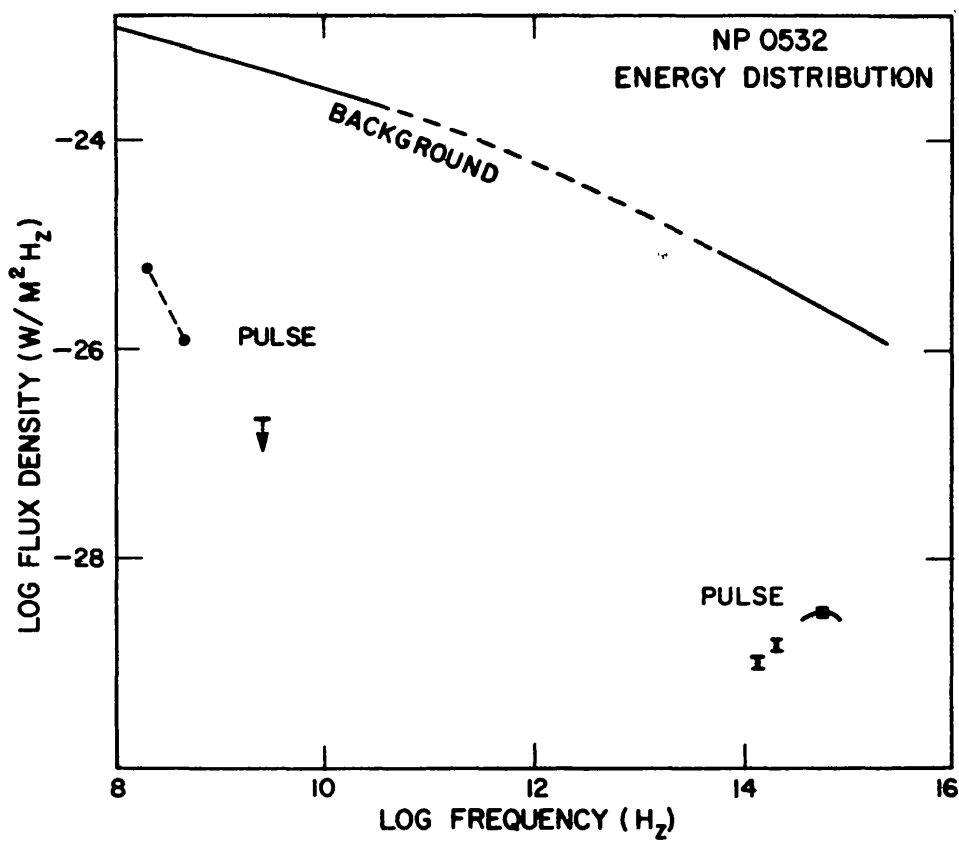

FIG. 3.-Radio- and optical-energy distribution of the main pulse of NP 0532. Optical points are those shown in Fig. 2. Two left-most points are measurements at 196 and $430 \mathrm{MHz}$ by Comella et al. (1969). Arrow shows an upper limit at $13 \mathrm{~cm}$ by Ekers and Moffet (1969). Most striking feature is lack of smoothness in the energy distribution, which has a large negative slope in the radio, a positive slope in the infrared, and an apparent peak in the visual. Data are presented as flux densities for direct comparison with the spectrum of the total radiation from the Crab Nebula, which is also shown. The latter is adapted from Fig. 2 of Becklin and Kleinmann (1968).

the observed energy at $2.2 \mu$ exceeds that which can be emitted by a $10000^{\circ} \mathrm{K}$ thermal source by a factor of approximately $10^{6}$.

All available radio and optical data on the energy distribution of the main pulse are plotted in Figure 3. The energy in the pulse at $2.2 \mu$ exceeds a linear extrapolation of the radio spectrum by a factor of almost $10^{8}$. The extrapolation from $10^{8}$ to $10^{14} \mathrm{~Hz}$ is clearly hazardous, but the change in the sign of the slope between the radio and infrared regions is certain. Although the amount of reddening assumed can change the size of the infrared slope, it cannot change the sign. There is no simple mechanism known to us, theoretically or observationally, which produces such an energy distribution. On the other hand, the similarity of the radio and optical pulses is remarkable. The radio data of Comella et al. (1969) show the same double pulses seen optically, with similar shapes, relative amplitudes, and separations of the pulses. Also, recent observations by Conklin et al. (1969) have established that the arrival times of the radio and optical pulses are 
the same, after corrections for interstellar dispersion, to within an observational uncertainty of the order of $6 \mathrm{msec}$. It appears, therefore, that the radio and optical pulses may be produced by mechanisms that differ in detail but are closely coupled, perhaps through a common pulsed energy source.

We are indebted to R. D. Ekers and A. T. Moffet for permission to include their unpublished 13-cm limit. We thank Gary Tuton and Judy Bennett for their help in obtaining and reducing the data. The synthesizer was borrowed from the Jet Propulsion Laboratory through M. S. Shumate. Discussions with J. B. Oke, R. Gomez, and R. Ekers were helpful.

\section{REFERENCES}

Becklin, E. E., and Kleinmann, D. E. 1968, Ap. J. (Letters), 152, L25.

Becklin, E. E., and Neugebauer, G. 1968, Ap. J., 151, 145.

Cocke, W. J., Disney, M. J., and Taylor, D. J. 1969, I.A.U. Circ. No. 2128.

Comella, J. M., Craft, H. D., Lovelace, R. V. E., and Sutton, J. M. 1969, Nature, 221, 453.

Conklin, E. K., Howard, H. T., Miller, J. S., and Wampler, E. J. 1969 (preprint).

Ekers, R. D., and Moffet, A. 1969 (private communication).

Johnson, H. L. 1964, Bol. Obs. Tonantzintla y Tacubaya, 3, 305.

- 1968, in Stars and Stellar Systems, Vol. 7, Nebulae and Interstellar Matter, ed. B. M. Middlehurst and L. H. Aller (Chicago: University of Chicago Press).

Lynds, R., Maran, S. P., and Trumbo, D. E. 1969, Ap. J. (Letters), 155, L121.

Nather, R. E., Warner, B., and Macfarlane, M. 1969, Nature, 221, 527.

Oke, J. B. 1969, Ap. J., 156, 491.

Westphal, J. A., Kristian, J., Snellen, G., Sandage, A., Schmidt, M., Oke, J. B., Neugebauer, G., and Becklin, E. E. 1969, Ap. J. (Letters), 155, L109. 